\title{
Genetic variant rs3750625 in the 3'UTR of ADRA2A affects the sleep quality of patients in the ICU by promoting miR-34a binding to ADRA2A
}

\author{
QINGPING HUANG $^{1 *}$, GANHAI CHEN $^{1 *}$, YANFANG HUANG ${ }^{2 *}$, JINTING LI $^{1}$, YANJING DING ${ }^{1}$, \\ SHUQING ZHANG ${ }^{2}, \mathrm{XIUWEN} \mathrm{CHI}^{3}$, QIONG XIE ${ }^{2}$, QINRONG NING ${ }^{4}$, LIUQIN XU $^{2}$ and JIANRONG ZHANG ${ }^{2}$ \\ ${ }^{1}$ ICU and ${ }^{2}$ Nursing Department, Dongguan Houjie Hospital, Dongguan, Guangdong 523945; ${ }^{3}$ School of Nursing, \\ Guangdong Medical University, Dongguan, Guangdong 523808; ${ }^{4}$ Department of General Surgery, \\ Dongguan Houjie Hospital, Dongguan, Guangdong 523945, P.R. China
}

Received April 14, 2018; Accepted May 10, 2019

DOI: $10.3892 / \mathrm{ijmm} .2020 .4456$

\begin{abstract}
Poor sleep is very common in patients in the ICU and hence, sleep quality is considered an important aspect of intensive care; however, the underlying mechanisms of poor sleep in patients in the ICU remain unknown. In this study, we aimed to explore the role of rs 3750625 , which is located in the 3'UTR of adrenoceptor alpha 2A (ADRA2A), in sleep quality. For this purpose, luciferase assay was conducted to investigate the association between miR-34a and ADRA2A, and the effect of rs3750625 on the binding affinity between miR-34a and ADRA2A was examined. RT-qPCR and western blot analysis were carried out to examine the regulatory association between miR-34a and ADRA2A. The differences in sleep time and efficiency were compared between groups carrying the $\mathrm{AC}$ and $\mathrm{CC}$ genotypes of rs 3750625 , respectively. According to the results from an online search, miR-34a could directly bind to the 3'UTR of ADRA2A, and such binding was confirmed by the observation that miR-34a inhibited the luciferase activity of major or minor ADRA2A 3'UTR in a dose-dependent manner in HCN-1A and U251 cells. In addition, the ADRA2A protein and mRNA levels in the HCN-1A and U251 cells were evidently decreased following transfection with miR-34a precursors. Notably, patients in the AC group exhibited a similar level of miR-34a mRNA expression compared with patients in the $\mathrm{CC}$ group; however, the ADRA2A mRNA and protein levels in the CC group were significantly increased in comparison with those in the
\end{abstract}

Correspondence to: Dr Jianrong Zhang, Nursing Department, Dongguan Houjie Hospital, 21 Hetian Road, Dongguan, Guangdong 523945, P.R. China

E-mail:medxicu@126.com

${ }^{*}$ Contributed equally

Key words: rs3750625, adrenoceptor alpha 2A, microRNA-34a, sleep quality, intensive care unit
AC group. In addition, the sleep time and sleep efficiency in the $\mathrm{CC}$ group were much higher than those in the AC group. Furthermore, the mean arterial pressure (MAP) values in both the $\mathrm{AC}$ and $\mathrm{CC}$ groups remained stable from 22:00 to 08:00, and the respiratory rates in both groups were quite similar. However, the heart rate of patients in the $\mathrm{CC}$ group was much lower than that of patients in the AC group. On the whole, the findings of this study suggest that the genetic variant rs 3750625 in the 3'UTR of ADRA2A affects the sleep quality of patients in the ICU by promoting the binding of miR-34a to ADRA2A, and hence it may serve as a novel biomarker for the prediction of the sleep quality of patients in the ICU.

\section{Introduction}

In recent years, the study of sleep disorders in critically ill patients has drawn increasing attention (1). In these patients, the amount of sleep is reduced and the pattern of sleep is fragmented without following the normal circadian rhythm. In addition, these patients have a higher proportion of sleep in the N1-N2 stages, whereas the amount of rapid eye movement (REM) in these patients is decreased. Since REM and sleep in the N3 stage play an important role in a wide range of physiological functions involving the immune, respiratory, endocrine, cardiovascular and central nervous systems, the lack of REM and N3 sleep in patients in the intensive care unit (ICU) significantly affects their recovery (1). For example, inadequate sleep has been shown to cause post-traumatic stress disorder, non-invasive ventilation failure and delirium (2-4).

It is known that $\alpha 2$ agonists can result in a loss of consciousness, a loss of righting reflex (LORR), presumed surrogate and sedation (5). On the other hand, the activity of locus coeruleus (LC) neurons is decreased during sleep and begins to increases before an individual wakes up, indicating that these neurons are associated with consciousness (6). Notably, although the selective stimulation of LC neurons induces waking, their inhibition cannot help sleep (7). In addition, in mice lacking the ability to produce noradrenalin (NA), an agonist of the adra $2 \mathrm{a}(\alpha 2 \mathrm{~A})$ receptor, the administration of dexmedetomidine can still result in LORR (5). Nevertheless, in the absence 
of its endogenous ligand, the response of $\alpha 2 \mathrm{~A}$-receptors can become hypersensitized in the long-term, making it difficult to interpret the results (5).

As a class of small and non-coding RNAs containing 19-25 nucleotides, microRNAs (miRNAs or miRs) have been shown to mediate the expression of a wide range of genes both in animals and plants (8). In addition, a number of miRNAs are involved in various cellular processes, such as metabolism, stress response, stem cell renewal, embryonic development, cell proliferation, apoptosis and differentiation (9). Given these critical functions of miRNAs, it is reasonable to expect that the abnormality of miRNA pathways and miRNA synthesis can result in several human diseases, including obesity, cardiovascular diseases, cancer, psoriasis, schizophrenia, chronic hepatitis, acquired immune deficiency syndrome (AIDS) and diabetes (10-15). In addition, single-nucleotide polymorphisms (SNPs) in the 3'untranslated region (3'UTR) of genes can alter the binding of miRNAs and affect the expression of target proteins, thus influencing the pathogenesis of a number of diseases (16). A previous study on healthy subjects demonstrated that a 3'UTR polymorphism in adrenoceptor alpha 2A (ADRA2A) mediates the onset of autonomic responses upon environmental and physiological stimulation, while the polymorphisms of ADRA2A can also be used to predict the responses to induced pain (17). In addition, the correlation between rs3750625, a 3'UTR SNP in ADRA2A, and acute musculoskeletal pain (MSP) has been evaluated among individuals experiencing sexual assault and motor vehicle collision (MVC) $(18,19)$. Furthermore, results of bioinformatics analyses have suggested that rs3750625 is located in the seed binding region of miR-34a, which is known to mediate stress and pain (20).

ADRA2A has been reported to be associated with sleep quality (21). Since miR-34a acts as a direct regulator of ARDA2A, the presence of rs3750625 has been shown to interrupt the interaction between miR-34a and ADRA2A $(18,22)$. Therefore, in this study, we evaluated the regulatory asociation between miR-34a and ARDA2A, as well as the association between rs3750625 and sleep quality in patients in the ICU.

\section{Materials and methods}

Collection of blood samples. The Human Research Ethics Committees of Dongguan Houjie Hospital approved this research. Written informed consent was obtained from all patients or their first-degree relatives prior to the onset of this research. The research process was in conformity with the latest version of Declaration of Helsinki. In this study, peripheral blood samples were collected from 38 subjects (the ethnicity of all participants was Han; sex: Female, 18 male, 20 mean age, $50 \pm 0.6$ years old) enrolled from patients who stay in ICU for at least 3 days. The inclusion criteria were the following: i) An age between 18 and 65 years; ii) a stay in the ICU of at least 3 days, but the use of mechanical ventilation not required; iii) stable hemodynamic parameters without sedative treatment. The exclusion criteria were the following: i) Patients diagnosed with hypoxic-ischemic encephalopathy; ii) patients diagnosed with psychiatric disorders; iii) patients diagnosed with hemodynamic instability; iv) patients diagnosed with severe abnormalities in the cardiac conduction system; v) patients diagnosed with ventricular dysfunction; vi) patients diagnosed with liver failure and a bilirubin level $>100 \mathrm{mmol} / \mathrm{l}$; vii) patients diagnosed with neurological injury or diseases that may influence sleep quality; viii) patients with a scale of Glasgow Coma score of $<11$; and ix) patients with an acute physiology score portion of the Acute physiology and Chronic Health evaluation II (APACHe II) score of $>15$.

Genotyping. Genomic DNA was isolated from abdomen tissue samples using a DNA extraction kit (Shunhua Bioengineer Co. Ltd.) and amplified using PCR. An ExoSAP-IT purification kit (USB) was utilized to purify the PCR products, which were then sent to a core facility for sequencing.

Sleep quality measurement. During the entire course of this study, the light in the ward was maintained at a minimum level so as to not affect the sleep of the subjects. For subjects in the 'no sedation' group of this study, they had to be removed from the study if they suffered from sleep disorders and required sedation. For all the remaining subjects, a measurement of bispectral index (BIS) was carried out by recording their sleep time and sleep depth continuously using a Covidien BIS VISTA monitor. In addition, the level of sedation (if applicable) and the depth of sleep were graded based on the BIS index, which scored EEG signals from 0 to 100. A score of 65-85 indicated a patient was in sedation or sleep, while a score of 85 to 100 suggested the patient was awake. In addition, the Richmond Agitation-Sedation Scale (RASS) score (23), the duration of consciousness and sleep, blood pressure, heart rate, respiratory rate, oxyhemoglobin saturation, and the duration of total sleep of each subject were examined every $2 \mathrm{~h}$. The ratio between the duration of effective sleep and the duration of total sleep was defined as sleep efficiency.

$R N A$ isolation and reverse transcription-quantitative $P C R$ (RT-qPCR). TRIzol reagent (Invitrogen; Thermo Fisher Scientific) was utilized to extract total RNA from HCN-1A and U251 cells and blood samples. The quality of the RNA was determined by measuring its absorbance with a NanoDrop 1000 spectrophotometer (Thermo Fisher Scientific). A Sprint Power Script Pre Primed Single Shots kit (Clontech) was utilized to synthesize first-strand ADRA2A cDNA from total RNA using the following reaction conditions: 5 cycles of $30 \mathrm{sec}$ at $94^{\circ} \mathrm{C}$, $30 \mathrm{sec}$ at $60^{\circ} \mathrm{C}$ and $60 \mathrm{sec}$ at $68^{\circ} \mathrm{C}$. A TaqMan hsa-miR-34a amplification kit (Applied Biosystems) was used to amplify the cDNA, which was then measured on an Applied Biosystems Real-Time PCR System (Applied Biosystems). The real-time PCR reaction mixture contained $4.0 \mu \mathrm{l}$ (a total of $40 \mathrm{ng}$ ) of ADRA2A cDNA, $10 \mu \mathrm{l}$ of $2 \mathrm{X}$ gene expression master mix, $1 \mu \mathrm{l}$ of 20X Gene Expression Assay buffer and $5 \mu 1$ of RNase-free water. The 3'UTR of ADRA2A was amplified with primers, including ATGATGCTCGAGACTCAGAAACCCGGGCGC (forward) and ATGATGAATTCCCATAAAATCAGATGT TCCCAGAG (reverse). GAPDH and U6 were used as internal controls to normalize the relative expression of miR-34a and ADRA2A, respectively. The reactive expression of miR-34a and ADRA2A mRNA was calculated using the $2^{-\Delta \Delta \mathrm{Cq}}$ method (24). Each experiment was carried out at least 3 times.

Cells, cell culture and transfection. The HCN-1A and U251 cell lines were obtained from the American Type Culture 
Human ADRA2A 5' -AAAATGCTAAGGGCAGCCCTGCCT- $3^{\prime}$

Dog Adra2a 5' -CAAATGCTCAGGGCAGCCCTGCCC- 3' Mouse Adra2a 5' -AAATCATTCAGGGCGGTCCTGCCT- 3'

Elephant Adra2a 5' -CAAACGCTAGGGGCAGCCCTGCCT- 3'

Chimpanzee Adra2a 5' -AAAATGCTAAGGGCAGCCCTGCCT- 3'

Rhesus monkey Adra2a 5' -AACCCACTCAGGGCAGCCCTGACT- 3' Rat Adra2a 5' -AAATCATTCAGGGCAGCCCTGCCT- 3'

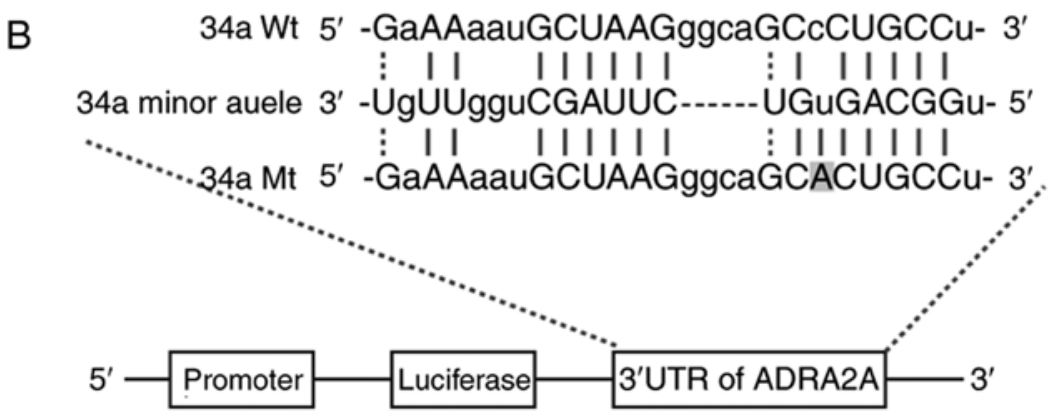

Figure 1. ADRA2A rs3750625 in the seed region containing putative binding site of miR-34a. (A) 'Seed sequence' in the 3' UTR of ADRA2A was highly conserved among species. (B) Single site or 'seed sequence' in ADRA2A 3'UTR were mutated, and major, minor or mutant ADRA2A 3'UTR were inserted into pGL3 vector immediately located downstream of luciferase gene. ADRA2A, adrenoceptor alpha 2A.

Collection (ATCC). For transfection experiments, the cells were seeded in 24 -well plates and cultured for $24 \mathrm{~h}$ at $37^{\circ} \mathrm{C}$ and $5 \% \mathrm{CO}_{2}$ in a RPMI medium containing $10 \%$ heat-inactivated fetal bovine serum (FBS), $50 \mathrm{U} / \mathrm{ml}$ streptomycin/penicillin and $2 \mathrm{mM}$ glutamine (Euroclone). When the cells reached 50-80\% confluence, they were transfected with $30 \mathrm{nM}$ miR-34a precursors, ADRA2A siRNA or a scramble control (RiboBio) using Lipofectamine 2000 (Invitrogen; Thermo Fisher Scientific). Subsequent experiments were accordingly performed after $48 \mathrm{~h}$ of transfection. Each experiment was performed at least 3 times.

Luciferase assay. The 3'-UTR of ADRA2A, which was found to contain the putative binding site of miR-34a by use of online database miRDB (http://mirdb.org/), was amplified using PCR. Subsequently, the PCR products (the major allele ADRA2A 3'UTR) were cloned into a pGL3 vector (Promega) and the correct sequence of the construct was confirmed by direct sequencing. In addition, a specific single-base mutation in ADRA2A 3'UTR (the minor allele ADRA2A 3'UTR), as well as a mutant ADRA2A 3'UTR was produced using a QuikChange ${ }^{\circledast}$ Lightning Site-Directed Mutagenesis kit (Agilent Technologies) and primers, including GCTAAG GGCAGCACTGCCTGCCCTC (forward) and GAGGGC AGGCAGTGCTGCCCTTAGC (reverse). Subsequently, both minor allele ADRA2A 3'UTR and mutant ADRA2A 3'UTR were inserted into pGL3 vectors (Promega), respectively, and the correct sequences of these constructs were also confirmed by direct sequencing. During the subsequent luciferase assay, $\mathrm{HCN}-1 \mathrm{~A}$ and U251 cells were co-transfected with $0.4 \mu \mathrm{g}$ of Firefly luciferase reporter constructs containing the major, minor or mutant ADRA2A 3'UTR, $0.02 \mu \mathrm{g}$ of control vector containing a Renilla luciferase (Promega), and various concentrations of miR-34a precursors using Lipofectamine 2000 (Invitrogen; Thermo Fisher Scientific). At $48 \mathrm{~h}$ post-transfection, a Dual-Luciferase Reporter Assay System (Promega) was utilized to determine the luciferase activity of Firefly luciferase, which was then normalized to the luciferase activity of Renilla luciferase to obtain the final results. All tests were performed in triplicate.

Western blot analysis. RIPA buffer (Invitrogen; Thermo Fisher Scientific) supplemented with a protease inhibitor cocktail and phosphatase (Roche Diagnostics) was used to extract the total protein from HCN-1A and U251 cells. The lysate was then boiled for $10 \mathrm{~min}$ in 2-mercaptoethanol and 10\% SDS-PAGE was used to separate the proteins. Subsequently, the proteins were transferred onto a nitrocellulose membrane (Amersham Biosciences, Piscataway, NJ) at $90 \mathrm{~V}$ for $2 \mathrm{~h}$. In the next step, the membrane was blocked at room temperature for $60 \mathrm{~min}$ in TBS (Tris-buffered saline) containing Tween-20 (TBST) and $5 \%$ non-fat milk, followed by incubation at $37^{\circ} \mathrm{C}$ for 120 min with goat anti-ADRA2A (ab45871, 1:5,000 dilution, Abcam) or anti- $\beta$-actin primary antibodies (4970s, 1:9,000 dilution, Cell Signaling Technologies). Subsequently, the membrane was washed and incubated at room temperature for $2 \mathrm{~h}$ with horseradish peroxidase (HRP)-conjugated anti-goat secondary antibodies (ab6721, 1:13,000 dilution, Abcam ). A Western Lightning Enhanced Chemiluminescence solution (Perkin Elmer) was used to detect the protein signals, which were acquired and analyzed using ImageJ software (NIH) to obtain the density of each protein band. All experiments were performed 3 times.

Statistical analysis. The data were analyzed by non-parametric tests and the significance of different variables was compared using one-way ANOVA, followed by Dunnett's test as a post hoc test. All continuous variables were presented as medians and a P-value of $<0.05$ was considered to indicate a statistically significant difference. All statistical analyses were carried out using SPSS 18.0 software (SPSS, Inc.). 

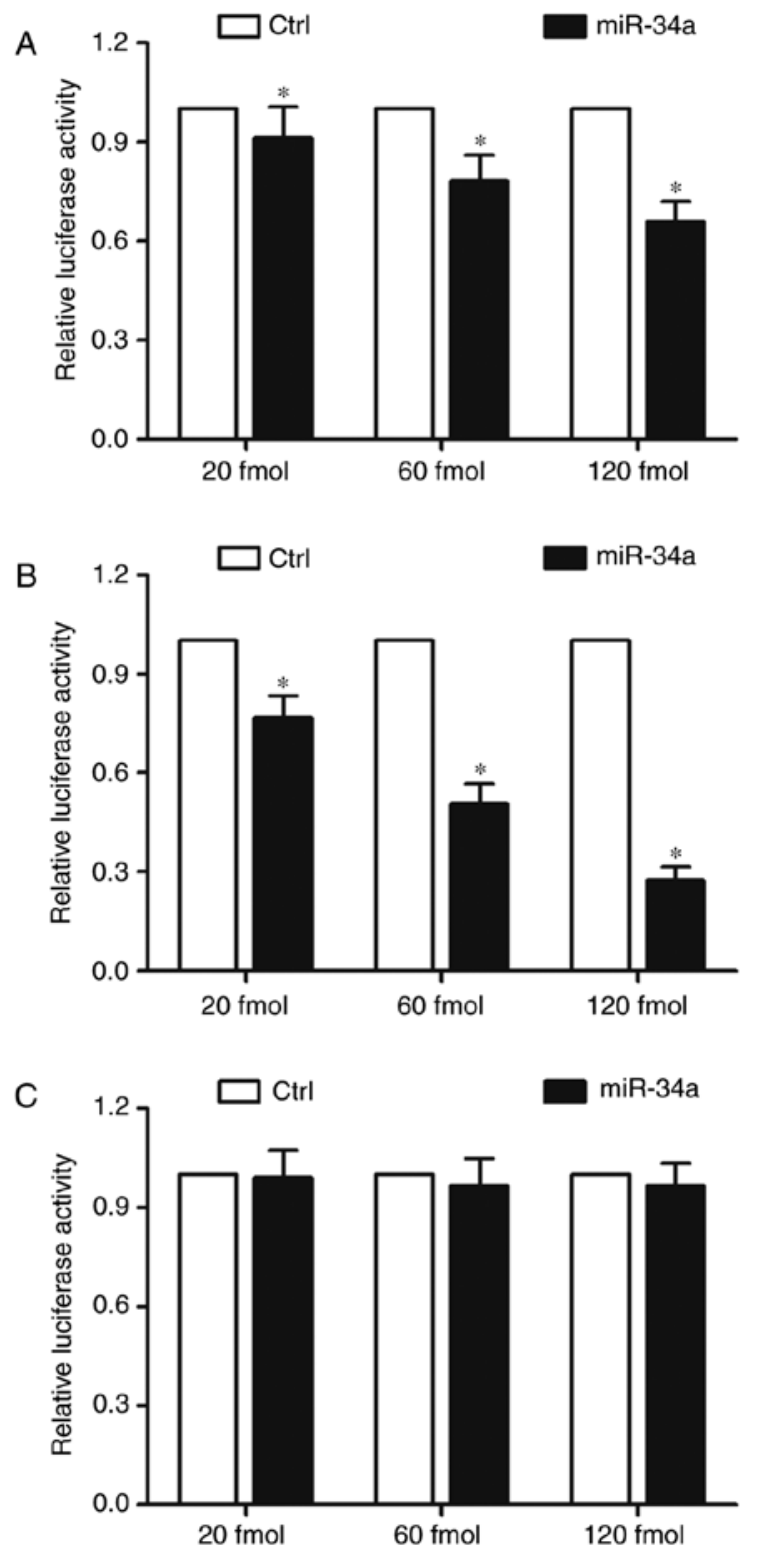

Figure 2. rs3750625 allele influences miR-34a binding in HCN-1A cells. (A) miR-34a mimic reduced the luciferase activity of major ADRA2A 3'UTR in a dose-dependent manner compared with the control. (B) miR-34a mimic dose-dependently inhibited the luciferase activity of minor ADRA2A 3'UTR compared with the control. (C) miR-34a mimic had no effect on the luciferase activity of mutant ADRA2A 3'UTR compared with the controls. ${ }^{*} \mathrm{P}<0.05$ compared with the control. ADRA2A, adrenoceptor alpha $2 \mathrm{~A}$.

\section{Results}

rs3750625 is located in the seed region of ADRA2A containing a putative binding site for miR-34a. According to the results of in silicon analysis by the utilization of miRdSNP (http://mirdsnp. ccr.buffalo.edu/), rs3750625 was located in the seed region of ADRA2A containing a putative binding site for miR-34a. In addition, A allele, the minor allele in rs 3750625 polymorphism, could increase the binding affinity between ADRA2A and miR-34a by creating an A-U base pair. Furthermore, the 'seed sequence' in the 3'UTR of ADRA2A was highly conserved among different species (Fig. 1A), indicating that such sequence may play a very important role. As shown in Fig. 1B, the binding of miR-34a to ADRA2A was successful when the major allele
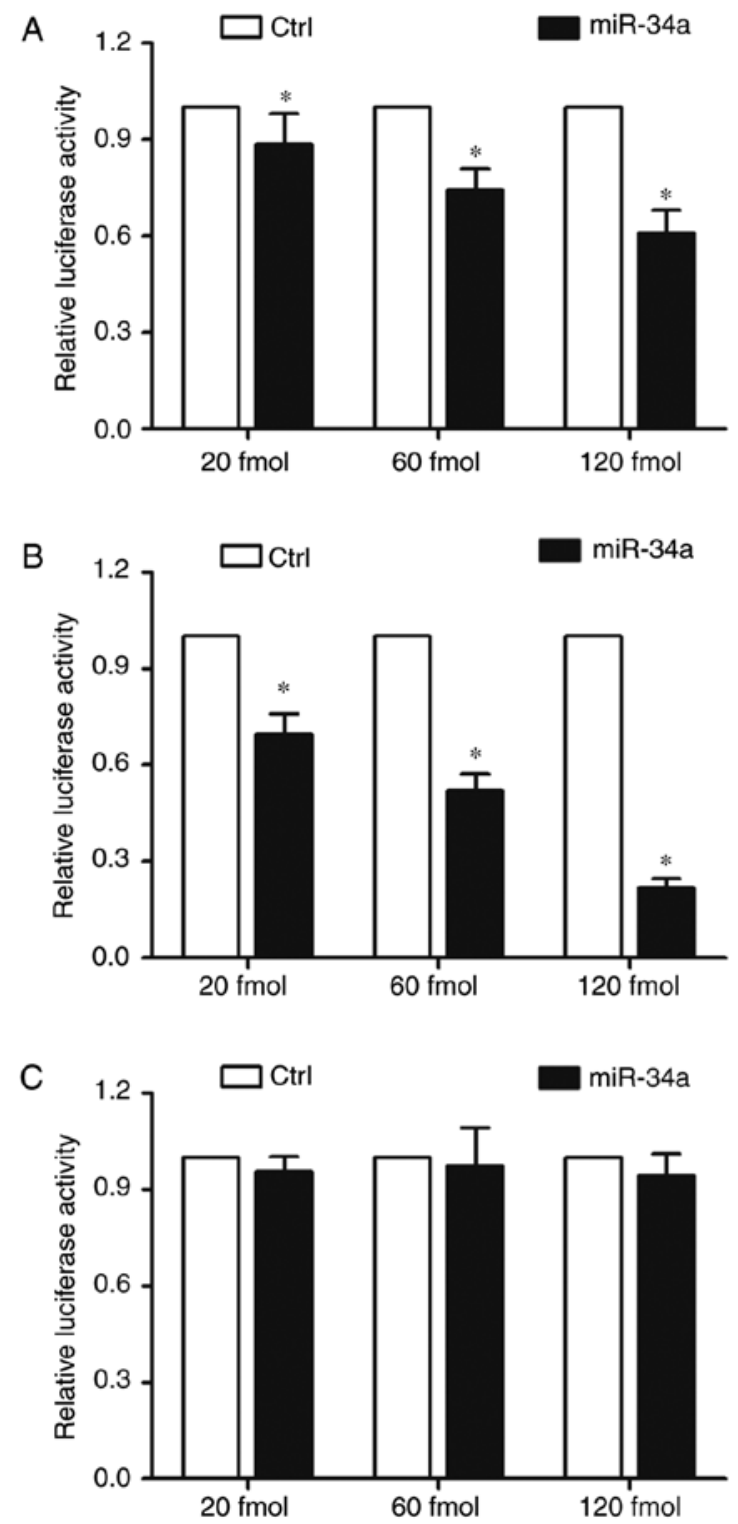

Figure 3. rs 3750625 allele influences miR-34a binding in U251 cells. (A) miR-34a mimic reduced the luciferase activity of the major ADRA2A 3'UTR in a dose-dependent manner compared with the control. (B) miR-34a mimic dose-dependently inhibited the luciferase activity of the minor ADRA2A 3'UTR compared with the control. (C) miR-34a mimic had no effect on the luciferase activity of mutant ADRA2A 3'UTR compared with the control. ${ }^{*} \mathrm{P}<0.05$ compared with the control. ADRA2A, adrenoceptor alpha $2 \mathrm{~A}$.

of ADRA2A was present; however, the mutation in ADRA2A 3'UTR hindered such binding.

rs3750625 allele affects miR-34a binding. According to our results from an online search upon miRDB, miR-34a could directly target ADRA2A 3'UTR (Fig. 1B). To further confirm the interaction between miR-34a and ADRA2A, the major, minor or mutant 3'UTR of ADRA2A was inserted into a pGL3 vector immediately downstream of the luciferase reporter gene (Fig. 1B), followed by the co-transfection of HCN-1A and U251 cells with these luciferase reporter constructs and various concentrations of the miR-34a mimics $(20 \mathrm{fmol}$, $60 \mathrm{fmol}$ and $120 \mathrm{fmol}$ ). As shown in Figs. 2A and 3A, transfection with miR-34a mimics reduced the luciferase activity 
A

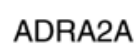

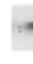

$\beta$-actin
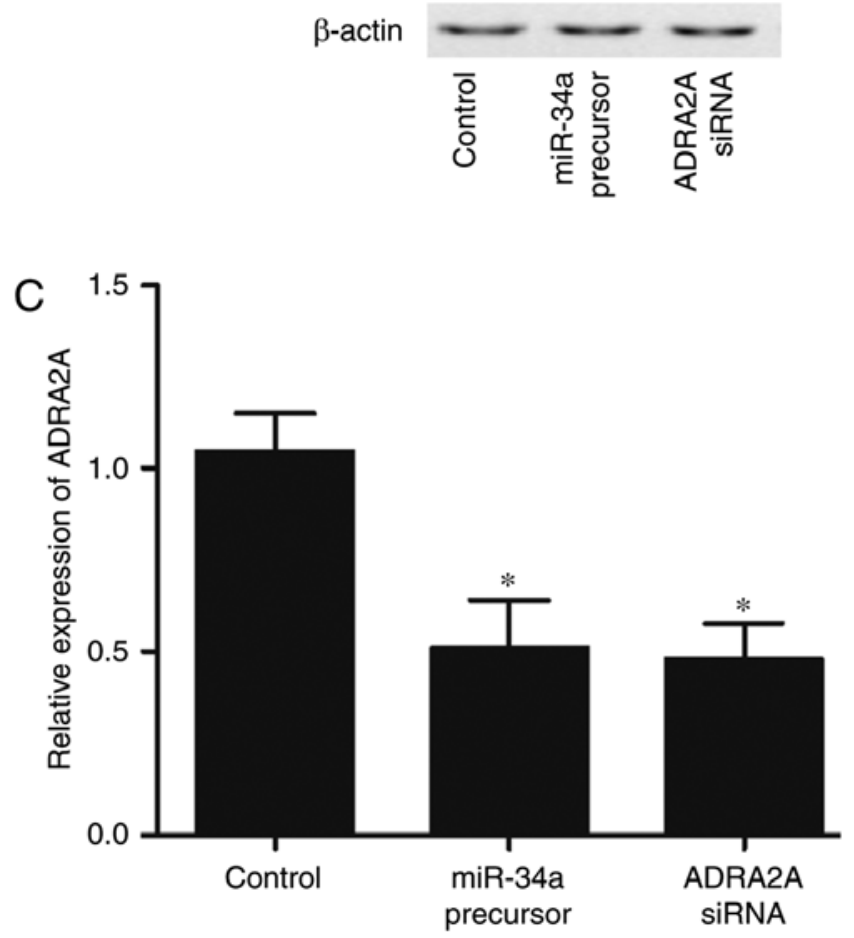

B

ADRA2A
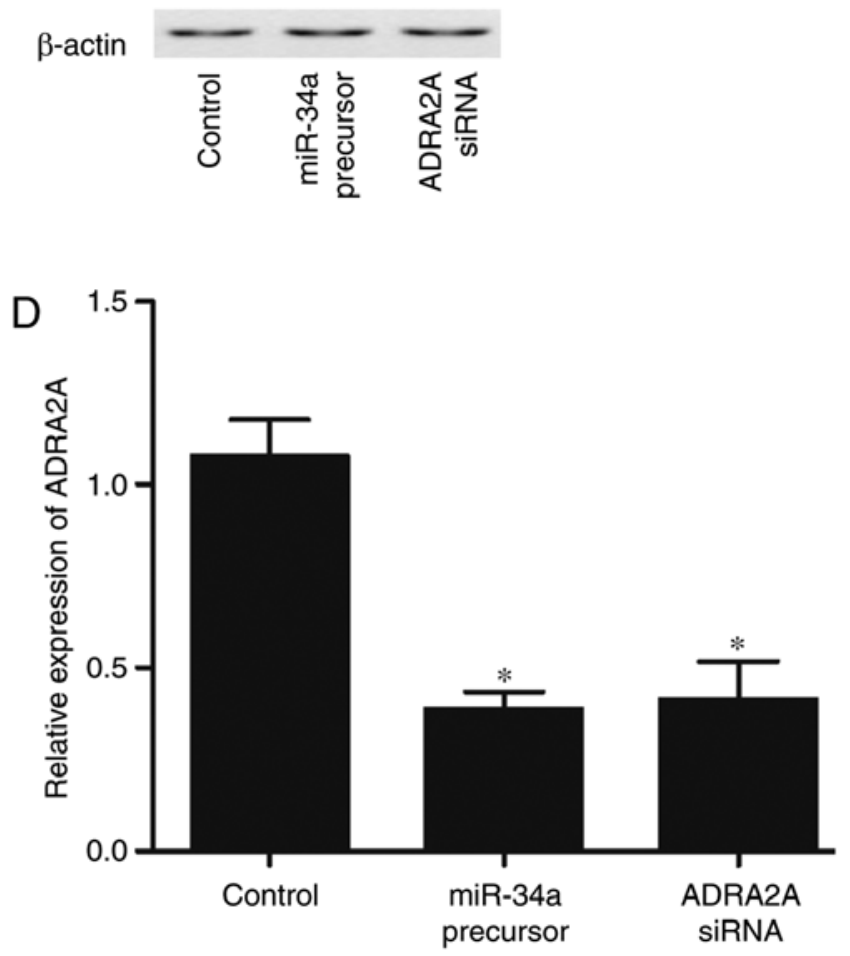

Figure 4. miR-34a suppresses ADRA2A expression. (A) ADRA2A protein level in HCN-1A cells transfected with miR-34a mimic was much lower than that of the control. (B) U251 cells transfected with miR-34a mimic exhibited a lower protein level of ADRA2A compared with the control. (C) ADRA2A mRNA level in HCN-1A cells transfected with miR-34a mimic was much lower than that of the control. (D) U251 cells transfected with miR-34a mimic exhibited a lower mRNA level of ADRA2A compared with the control. ${ }^{*} \mathrm{P}<0.05$ compared with the control. ADRA2A, adrenoceptor alpha $2 \mathrm{~A}$.

of the cells transfected with the major ADRA2A 3'UTR, and such a decrease in luciferase activity was dose-dependent. Furthermore, the luciferase activity in the cells transfected with the major ADRA2A 3'UTR decreased in a stepwise manner at an increasing concentration of miR-34a mimics (Figs. 2B and 3B). Notably, when the concentration of miR-34a remained the same, the inhibitory effect of miR-34a on the luciferase activity of the cells transfected with the minor ADRA2A 3'UTR was much stronger than that of the cells transfected with the major ADRA2A 3'UTR. As expected, miR-34a mimics exerted no effect on the luciferase activity of the cells transfected with the mutant ADRA2A 3'UTR (Figs. 2C and 3C), suggesting that the A allele located in rs3750625 increased the binding affinity between ADRA2A and miR-34a, thus reducing the expression of ADRA2A.

miR-34a suppresses ADRA2A expression. To further confirm whether miR-34a inhibits ADRA2A expression, the HCN-1A and U251 cells were transfected with miR-34a precursor or ADRA2A siRNA, as shown in Fig. 4. Following transfection with miR-34a precursor or ADRA2A siRNA, both the HCN-1A (Fig. 4A and C) and U251 (Fig. 4B and D) cells exhibited a decreased protein and mRNA expression of ADRA2A.

miR-34a and ADRA2A are differentially expressed in the different groups. The 38 subjects in this study were divided into 2 groups, i.e., the $\mathrm{CC}(\mathrm{n}=23)$ and $\mathrm{AC}(\mathrm{n}=15)$ groups, according to their genotype of rs3750625. Subsequently, RT-qPCR and western blot analysis were performed to measure the levels of miR-34a, ADRA2A mRNA and ADRA2A protein in these 2 groups. As shown in Fig. 5A, the level of miR-34a in the $\mathrm{CC}$ group was similar to that in the AC group; however, the protein and mRNA level of ADRA2A in the CC group was much higher than that in the AC group (Fig. 5B and C).

Determination of sleep quality in different groups. The duration of sleep and the depth of sleep in the 2 groups were continuously monitored and recorded using a Covidien BIS VISTA machine. As shown in Fig. 6, sleep time (Fig. 6A) and sleep efficiency (Fig. 6B) in the CC group were much higher than those in the AC group, indicating that ADRA2A rs3750625 polymorphism was associated with the sleep quality of patients in the ICU.

Mean arterial pressure (MAP), heart rate and respiratory rate in the different groups. Compared to the baseline values, the value of MAP In the AC group was similar to that in the $\mathrm{CC}$ group. In fact, the MAP values in both AC and CC groups remained stable from 22:00 to 07:00, measured every $1.5 \mathrm{~h}$ (Fig. 7A). In addition, a lower heart rate was observed among patients in the CC group as compared to those in the AC group (Fig. 7B). Furthermore, compared to the baseline values, the subjects in the $\mathrm{CC}$ group exhibited a significantly reduced heart rate tendency (Fig. 7B). As shown in Fig. 7C, no obvious difference was observed between the $\mathrm{AC}$ and $\mathrm{CC}$ groups in terms of respiratory rate. 

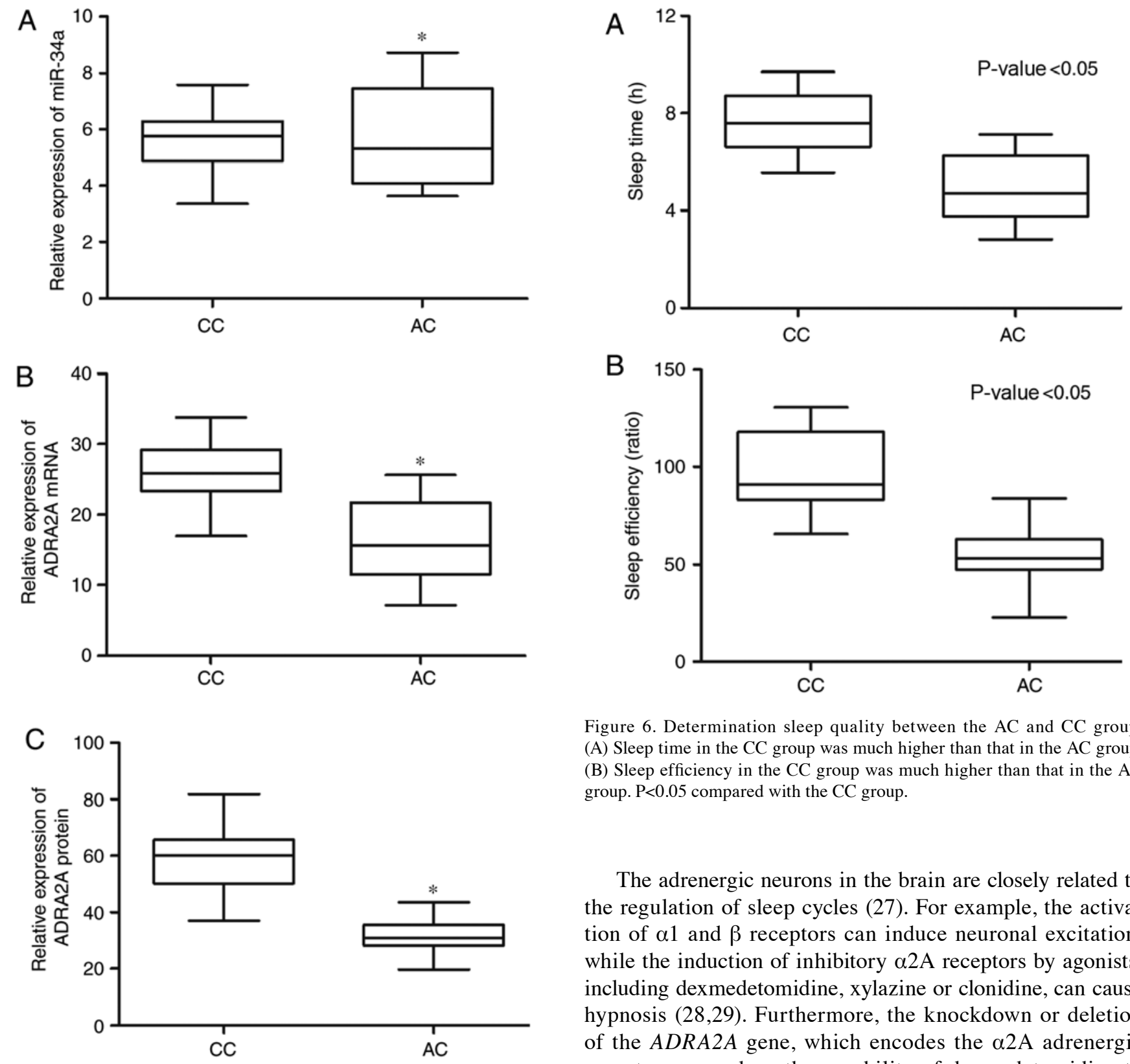

Figure 5. miR-34a and ADRA2A are differentially expressed in the different groups. (A) miR-34a mRNA in AC group exhibited no obvious difference with that in the CC group. (B) ADRA2A mRNA was highly expressed in the $\mathrm{CC}$ group compared with the $\mathrm{AC}$ group. (C) ADRA2A protein level in the $\mathrm{CC}$ group was much higher than that in the $\mathrm{AC}$ group. ${ }^{*} \mathrm{P}<0.05$ compared with the control. ADRA2A, adrenoceptor alpha 2A.

\section{Discussion}

Poor sleep is very common among patients in the ICU and hence, sleep quality is considered an important aspect of intensive care (25). In fact, patients in the ICU spend approximately $50 \%$ of their total sleeping time during the daytime, with a significantly reduced or missing circadian rhythm (26). Furthermore, inadequate sleep may cause extended hospitalization, as well as an extended stay in the ICU. In severe cases, inadequate sleep can trigger long-term cognitive impairment and delirium (27). Therefore, sedation is critical for a successful treatment of patients in the ICU as it can reduce pain, relieve anxiety and decrease stress.

Figure 6. Determination sleep quality between the AC and CC group. (A) Sleep time in the $\mathrm{CC}$ group was much higher than that in the $\mathrm{AC}$ group. (B) Sleep efficiency in the CC group was much higher than that in the AC group. $\mathrm{P}<0.05$ compared with the $\mathrm{CC}$ group.

The adrenergic neurons in the brain are closely related to the regulation of sleep cycles (27). For example, the activation of $\alpha 1$ and $\beta$ receptors can induce neuronal excitation, while the induction of inhibitory $\alpha 2 \mathrm{~A}$ receptors by agonists, including dexmedetomidine, xylazine or clonidine, can cause hypnosis $(28,29)$. Furthermore, the knockdown or deletion of the ADRA2A gene, which encodes the $\alpha 2 \mathrm{~A}$ adrenergic receptor, can reduce the capability of dexmedetomidine to trigger LORR (30). Since the location of ADRA2A expression in the brain is highly restricted, it is possible to not only identify which downstream pathways are used by dexmedetomidine to trigger sleep, but also determine which pathways can inhibit sleep, thus allowing us to gain insight into natural sleep circuitry $(31,32)$.

The ADRA2A gene is found in chromosome 10q24-q26 and is located in a linkage region to T2DM (33). It was shown previously that $A D R A 2 A$ is involved in the function of $\beta$ cells (34). Animal models have been used to study the function of miRNAs in the regulation of sleep. For example, sleep deprivation can lead to a significant change in miRNA profiles in rat adipose tissues and brain, and such a change is independent of the plasma level of corticosterone (35). In addition, sleep in experimental animals can be altered by the cortical or intraventricular injection of miRNAs and specific anti-miRNAs into the brain, as suggested by the slow wave activity on electroencephalographic (EEG) (36). The role of miRNAs in the control of sleep quality may be mediated by their ability to regulate the expression of sleep associated 

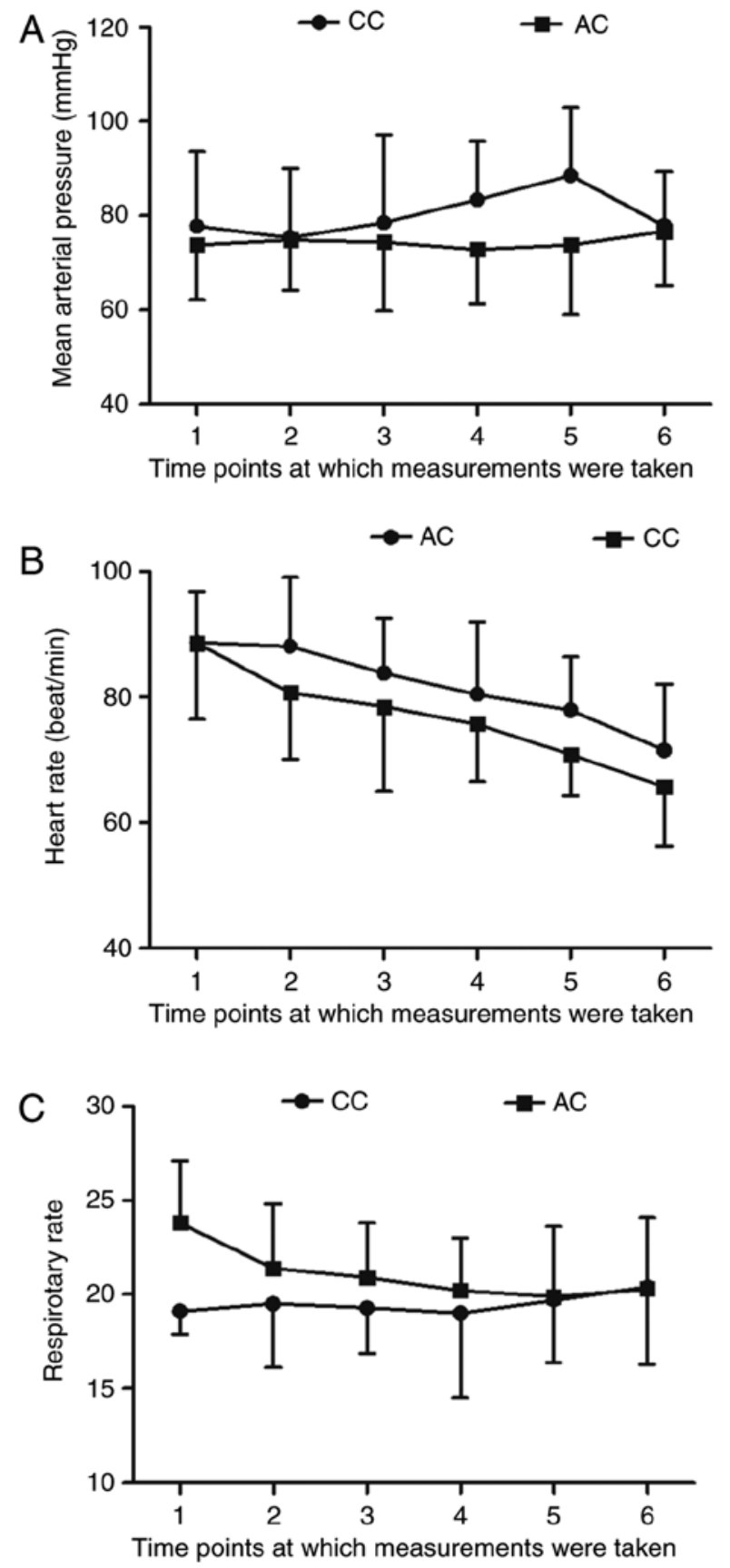

Figure 7. (A) The trend of mean arterial pressure (MAP) between the AC and $\mathrm{CC}$ groups. (B) The heart rate trend between the $\mathrm{AC}$ and $\mathrm{CC}$ groups. (C) The trend of respiratory rate between the AC and CC groups. Note that the $\mathrm{x}$ axes in the figure indicate the time points at which measurements were taken. Measurements were taken over a 9-h time period from 22:00-07:00. Measurements were taken every $1.5 \mathrm{~h}$. The time points labeled 1-6 on the $\mathrm{x}$ axes indicate the 6 time points at which measurements were taken.

genes. Recently, the full 3'UTR of ADRA2A was cloned into a luciferase reporter plasmid downstream of a Firefly luciferase reporter gene, and the luciferase assay results revealed a potential location for miR-34a binding, which in turn led to the reduction in luciferase activity in a dose-dependent manner $(18,37)$. In this study, we searched an online database and found that miR-34a could directly bind to the 3'UTR of ADRA2A. In addition, a single site or a 'seed sequence' in ADRA2A 3'UTR were mutated in this study to generate minor or mutant ADRA2A 3'UTR, which were then inserted into pGL3 vectors to generate different luciferase constructs. Furthermore, the above-mentioned luciferase constructs were transfected into the $\mathrm{HCN}-1 \mathrm{~A}$ and $\mathrm{U} 251$ cells, and the results revealed that miR-34a reduced the luciferase activity of the cells transfected with the major or minor ADRA2A 3'UTR in a dose-dependent manner. As expected, miR-34a had no effect on the luciferase activity of the cells transfected with the mutant ADRA2A 3'UTR.

It was previously suggested that, following sexual assaultor MVC-associated trauma, subjects harboring the rs3750625 minor allele may show a 1-point increase (on a scale of 0-10) during acute MSP. This may be caused by a more efficient binding between ADRA2A 3'UTR and miR-34a in the presence of the minor allele. Such results are consistent with the role of miR-34a in the regulation of stress, indicating that the symptoms of stress may be caused by the activation of physiologic systems that also promote miR-34a expression and increase the effect of rs3750625 on stress $(18,38)$. In this study, we recruited 38 patients in the ICU and divided them into 2 groups, i.e., the $\mathrm{CC}(\mathrm{n}=32)$ and $\mathrm{AC}(\mathrm{n}=6)$ groups, according to their genotypes of $\mathrm{rs} 3750625$. The results revealed that miR-34a expression in the CC group was comparable to that in the AC group, whereas the mRNA and protein level of ADRA2A was significantly higher in the CC group compared to that in the AA group. In addition, we found that the sleep time and sleep efficiency in the $\mathrm{CC}$ group were much higher than those in the AC group. Furthermore, we revealed that the MAP value in both the AC and $\mathrm{CC}$ groups remained stable from 22:00 to 08:00, and their respiratory rates were also similar. However, a lower heart rate was observed in the patients from the CC group. Donello et al previously demonstrated that animals displayed acute hyperalgesia upon sound exposure if ADRA2A was absent (39). It has also been shown that, in individuals harboring the minor allele of rs3750625, an increased level of miR-34a binding, a reduced level of ADRA2A transcripts, and an increased level of acute MSP were observed in those suffering from trauma (18). rs3750625 has been previously shown to play a role in the postprandial gastric volume of obese or overweight European-Americans (22). It has also been demonstrated that rs3750625 is close to or within the seed region of 23 miRNAs, suggesting that rs3750625 may increase the expression of ADRA2A by affecting miRNA binding (18). In a recent study, Linnstaedt et al demonstrated that rs3750625 further decreased ADRA2A expression by increasing miR-34a binding affinity, and hence reduced the risk of gestational diabetes mellitus (GDM) by increasing the release of insulin (18).

There are limitations to this study: Firstly, the sample size of this study was relatively small, and further studies with a larger sample size are warranted to confirm the conclusions of this study; secondly, further functional studies are warranted to confirm the conclusions of this study.

Taken together, it can be concluded that the SNP rs3750625 in the 3'UTR of ADRA2A affects the sleep quality of patients in the ICU in by increasing the binding of miR-34a to ADRA2A. In brief, the presence of the rs3750625 minor allele in the 3'UTR of ADRA2A increases the binding between miR-34a and ADRA2A, a direct target of miR-34a. As a result, by further inhibiting the expression of ADRA2A, the presence of the rs3750625 minor allele can affect the sleep quality of patients in the ICU. 


\section{Acknowledgements}

Not applicable.

\section{Funding}

No funding was received.

\section{Availability of data and materials}

All data generated or analyzed during this study are included in this published article or are available from the corresponding author on reasonable request.

\section{Authors' contributions}

$\mathrm{QH}$ and $\mathrm{JZ}$ were involved in the conception and design of the study. GC, YH and JL were involved in the literature collection. QH, GC, YD, SZ, XC, QX and LX were involved in data collection. YH, JL, QN and JZ were involved in data analysis and processing. QH, GC and $\mathrm{JZ}$ were involved in manuscript preparation. YH, JL, YD, SZ, XC, QX, QN and LX were involved in the final proofreading of the manuscript. All authors have read and approved the final manuscript.

\section{Ethics approval and consent to participate}

The Human Research Ethics Committees of Dongguan Houjie Hospital approved this research. Written informed consent was obtained from all patients or their first-degree relatives prior to the onset of this research. The research process was in conformity with the latest version of Declaration of Helsinki.

\section{Patient consent for publication}

Not applicable.

\section{Competing interests}

The authors declare that they have no competing interests.

\section{References}

1. Pisani MA, Friese RS, Gehlbach BK, Schwab RJ, Weinhouse GL and Jones SF: Sleep in the intensive care unit. Am J Respir Crit Care Med 191: 731-738, 2015.

2. Weinhouse GL, Schwab RJ, Watson PL, Patil N, Vaccaro B, Pandharipande $\mathrm{P}$ and Ely EW: Bench-to-bedside review: Delirium in ICU patients-importance of sleep deprivation. Crit Care 13: 234, 2009.

3. Roche Campo F, Drouot X, Thille AW, Galia F, Cabello B, d'Ortho MP and Brochard L: Poor sleep quality is associated with late noninvasive ventilation failure in patients with acute hypercapnic respiratory failure. Crit Care Med 38: 477-485, 2010

4. Wulff K, Gatti S, Wettstein JG and Foster RG: Sleep and circadian rhythm disruption in psychiatric and neurodegenerative disease. Nat Rev Neurosci 11: 589-599, 2010.

5. Sanders RD and Maze M: Noradrenergic trespass in anesthetic and sedative states. Anesthesiology 117: 945-947, 2012.

6. Takahashi K, Kayama Y, Lin JS and Sakai K: Locus coeruleus neuronal activity during the sleep-waking cycle in mice. Neuroscience 169: 1115-1126, 2010.

7. Carter ME, Yizhar O, Chikahisa S, Nguyen H, Adamantidis A, Nishino S, Deisseroth K and de Lecea L: Tuning arousa with optogenetic modulation of locus coeruleus neurons. Nat Neurosci 13: 1526-1533, 2010.
8. Lee RC, Feinbaum RL and Ambros V: The C. elegans heterochronic gene lin-4 encodes small RNAs with antisense complementarity to lin-14. Cell 75: 843-854, 1993.

9. Karp X and Ambros V: Developmental biology. Encountering microRNAs in cell fate signaling. Science 310: 1288-1289, 2005.

10. Weiler J, Hunziker J and Hall J: Anti-miRNA oligonucleotides (AMOs): Ammunition to target miRNAs implicated in human disease. Gene Ther 13: 496-502, 2006.

11. Latronico MV, Catalucci D and Condorelli G: Emerging role of microRNAs in cardiovascular biology. Circ Res 101: 1225-1236, 2007.

12. Lynam-Lennon N, Maher SG and Reynolds JV: The roles of microRNA in cancer and apoptosis. Biol Rev Camb Philos Soc 84: 55-71, 2009.

13. Sonkoly E, Wei T, Janson PC, Saaf A, Lundeberg L, TengvallLinder M, Norstedt G, Alenius H, Homey B, Scheynius A, et al: MicroRNAs: Novel regulators involved in the pathogenesis of psoriasis. PLoS One 2: e610, 2007.

14. Hansen T, Olsen L, Lindow M, Jakobsen KD, Ullum H, Jonsson E, Andreassen OA, Djurovic S, Melle I, Agartz I, et al: Brain expressed microRNAs implicated in schizophrenia etiology. PLoS One 2: e873, 2007.

15. Murakami Y, Kubo S, Tamori A, Itami S, Kawamura E, Iwaisako K, Ikeda K, Kawada N, Ochiya T and Taguchi YH: Comprehensive analysis of transcriptome and metabolome analysis in Intrahepatic Cholangiocarcinoma and Hepatocellular Carcinoma. Sci Rep 5: 16294, 2015.

16. Deveci M, Catalyurek UV and Toland AE: mrSNP: Software to detect SNP effects on microRNA binding. BMC Bioinformatics 15: 73, 2014.

17. Finley JC Jr, O'Leary M, Wester D, MacKenzie S, Shepard N, Farrow $S$ and Lockette W: A genetic polymorphism of the alpha2-adrenergic receptor increases autonomic responses to stress. J Appl Physiol 96: 2231-2239, 1985.

18. Linnstaedt SD, Walker MG, Riker KD, Nyland JE, Hu J, Rossi C, Swor RA, Jones JS, Diatchenko L, Bortsov AV, et al: Genetic variant rs3750625 in the 3'UTR of ADRA2A affects stress-dependent acute pain severity after trauma and alters a microRNA-34a regulatory site. Pain 158: 230-239, 2017.

19. Kohli U, Muszkat M, Sofowora GG, Harris PA, Friedman EA, Dupont WD, Scheinin M, Wood AJ, Stein CM and Kurnik D: Effects of variation in the human alpha2A- and alpha2C-adrenoceptor genes on cognitive tasks and pain perception. Eur J Pain 14: 154-159, 2010.

20. Haramati S, Navon I, Issler O, Ezra-Nevo G, Gil S, Zwang R, Hornstein E and Chen A: MicroRNA as repressors of stress-induced anxiety: The case of amygdalar miR-34. J Neurosci 31: 14191-14203, 2011.

21. Gelegen C, Gent TC, Ferretti V, Zhang Z, Yustos R, Lan F, Yang Q, Overington DW, Vyssotski AL, van Lith HA, et al: Staying awake-a genetic region that hinders $\alpha 2$ adrenergic receptor agonist-induced sleep. Eur J Neurosci 40: 2311-2319, 2014.

22. Papathanasopoulos A, Camilleri M, Carlson PJ, Vella A, Nord SJ, Burton DD, Odunsi ST and Zinsmeister AR: A preliminary candidate genotype-intermediate phenotype study of satiation and gastric motor function in obesity. Obesity (Silver Spring) 18: 1201-1211, 2010.

23. Sessler CN, Gosnell MS, Grap MJ, Brophy GM, O'Neal PV, Keane KA, Tesoro EP and Elswick RK: The richmond agitation-sedation scale: Validity and reliability in adult intensive care unit patients. Am J Respir Crit Care Med 166: 1338-1344, 2002.

24. Livak KJ and Schmittgen TD: Analysis of relative gene expression data using real-time quantitative PCR and the Delta Delta $\mathrm{C}(\mathrm{T}))$ method. Methods 25: 402-408, 2001.

25. Freedman NS, Gazendam J, Levan L, Pack AI and Schwab RJ: Abnormal sleep/wake cycles and the effect of environmental noise on sleep disruption in the intensive care unit. Am J Respir Crit Care Med 163: 451-457, 2001.

26. Parthasarathy $\mathrm{S}$ and Tobin MJ: Sleep in the intensive care unit. Intensive Care Med 30: 197-206, 2004.

27. Saper CB, Fuller PM, Pedersen NP, Lu J and Scammell TE: Sleep state switching. Neuron 68: 1023-1042, 2010.

28. Schmeichel BE and Berridge CW: Wake-promoting actions of noradrenergic $\alpha 1$ - and beta-receptors within the lateral hypothalamic area. Eur J Neurosci 37: 891-900, 2013.

29. Drew GM, Gower AJ and Marriott AS: Alpha 2-adrenoceptors mediate clonidine-induced sedation in the rat. Br J Pharmacol 67: 133-141, 1979. 
30. Lakhlani PP, MacMillan LB, Guo TZ, McCool BA,Lovinger DM, Maze $M$ and Limbird LE: Substitution of a mutant alpha2a-adrenergic receptor via 'hit and run' gene targeting reveals the role of this subtype in sedative, analgesic, and anesthetic-sparing responses in vivo. Proc Natl Acad Sci USA 94: 9950-9955, 1997.

31. Franks NP: General anaesthesia: From molecular targets to neuronal pathways of sleep and arousal. Nat Rev Neurosci 9: 370-386, 2008

32. Nicholas AP, Pieribone V and Hokfelt T: Distributions of mRNAs for alpha-2 adrenergic receptor subtypes in rat brain: An in situ hybridization study. J Comp Neurol 328: 575-594, 1993.

33. Duggirala R, Blangero J, Almasy L, Dyer TD, Williams KL, Leach RJ, O'Connell P and Stern MP: Linkage of type 2 diabetes mellitus and of age at onset to a genetic location on chromosome 10q in Mexican Americans. Am J Hum Genet 64: 1127-1140, 1999.

34. Boesgaard TW, Grarup N, Jorgensen T, Borch-Johnsen K, Hansen T, Pedersen O and Meta-Analysis of Glucose and Insulin-Related Trait Consortium (MAGIC): Variants at DGKB/TMEM195, ADRA2A, GLIS3 and C2CD4B loci are associated with reduced glucose-stimulated beta cell function in middle-aged Danish people. Diabetologia 53: 1647-1655, 2010.
35. Liu KX, Chen GP, Lin PL, Huang JC, Lin X, Qi JC and Lin QC: Detection and analysis of apoptosis- and autophagy-related miRNAs of mouse vascular endothelial cells in chronic intermittent hypoxia model. Life Sci 193: 194-199, 2018.

36. Borbely S, Vilagi I, Haraszti Z, Szalontai O, Hajnik T, Toth A and Detari L: Sleep deprivation decreases neuronal excitability and responsiveness in rats both in vivo and ex vivo. Brain Res Bull 137: 166-177, 2018.

37. Gottwein E and Cullen BR: A human herpesvirus microRNA inhibits p21 expression and attenuates p21-mediated cell cycle arrest. J Virol 84: 5229-5237, 2010.

38. Dias BG, Goodman JV, Ahluwalia R, Easton AE, Andero R and Ressler KJ: Amygdala-dependent fear memory consolidation via miR-34a and Notch signaling. Neuron 83: 906-918, 2014.

39. Donello JE, Guan Y, Tian M, Cheevers CV, Alcantara M, Cabrera S, Raja SN and Gil DW: A peripheral adrenoceptor-mediated sympathetic mechanism can transform stress-induced analgesia into hyperalgesia. Anesthesiology 114: 1403-1416, 2011. 